\title{
Immobilization of pharmaceuticals on mineral raw materials
}

\author{
AGNIESZKA SOLIŃSKA ${ }^{1}$, MATEUSZ SKALNY ${ }^{1}$ AND \\ TOMASZ BAJDA ${ }^{2}$
}

${ }^{1}$ AGH University of Science and Technology, Faculty of Geology, Geophysics and Enviromental Protection

${ }^{2}$ AGH University of Science and Technology

Presenting Author: asolinska@agh.edu.pl

The presence of pharmaceuticals in freshwater and groundwater pose a risk for human and animal health. Especially, big attention is focused on occurrence of distinct amounts of non-steroidal anti-inflammatory drugs (NSAIDs) and antibiotics. For the last years, their possibilities of immobilizations have been intensively studied. Nevertheless, adsorption method with applying relatively low-cost mineral materials might be promising solutions for effective removal of pharmaceuticals from water. Herein, we investigated lignite and fly ash in batch sorption experiments in order to remove drugs: ibuprofen and sulfamethoxazole. The chromatography analysis has clearly shown that those materials are capable of effective immobilization of pharmaceuticals. The sorbents were analyzed with X-ray diffraction (XRD), Fourier-transform infrared spectroscopy (FTIR) and scanning electron microscopy (SEM). The textural parameters were characterized based on data obtained from $\mathrm{N}_{2}$ isotherms (specific surface area, pore size distribution). The obtained results suggest that individual features of each sorption material, among others porosity, presence of significant amount of unburned coal and diverse functional groups combined with chemical properties of ibuprofen and sulfamethoxazole lead to immobilization of these emerging contaminants from polluted water.

This work has been partly supported by the EU Project POWR.03.02.00-00-I038/16-00. 